\title{
Comment freiner durablement la hausse des primes
}

\section{Yvonne Gilli}

Dre méd., présidente de la FMH

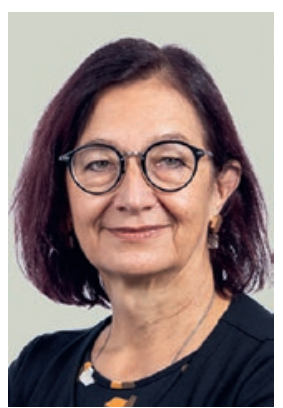

Les nouvelles à l'approche de l'annonce des primes 2022 sont plutôt optimistes. Après les faibles augmentations de ces dernières années $(1,2 \%, 0,2 \%$ et $0,5 \%)$ [1], la hausse devrait rester très modérée $(0,8 \%)$ [2]. Mais cette année aussi, l'alarmisme viendra ternir cette note positive. On entend notamment que c'est la dissolution de réserves qui tient les primes à un niveau «artificiellement bas». Cette affirmation, assurément trompeuse, rappelle l'importance de se pencher sur son véritable contenu: de facto, l'évolution des primes n'a jamais reflété directement l'évolution des coûts. D'une part, les primes approuvées par l'OFSP se réfèrent à chaque fois à des coûts pro-

\section{L'évolution des primes n’a jamais reflété direc- tement l'évolution des coûts.}

nostiqués pour l'année suivante. Elles sont plutôt fixées à un niveau trop élevé en raison des exigences légales et servent, comme l'indique l'OFSP [3], à la «constitution, nécessaire, de réserves». Durant 23 des 24 années écoulées depuis l'introduction de l'assurance obligatoire, le montant des primes payées a été supérieur aux prestations nettes auxquelles elles donnaient droit, avec des différences particulièrement marquées entre 2017 et 2019 [4]. Ces réserves qu'il s'agit aujourd'hui de réduire existent parce que les assurés ont payé davantage que les prestations dont ils ont bénéficié. On peut donc dire que les primes étaient alors à un niveau «artificiellement élevé».

Point plus important encore. Aujourd'hui, les primes financent $38 \%$ de notre système de santé, contre $30 \%$ seulement au moment de l'introduction de la LAMal en 1996 [5]. Les coûts financés par les primes ont par conséquent beaucoup plus augmenté que les coûts totaux, respectivement de $175 \%$ contre $117 \%$ [5]. On ne parle donc pas ici d'une simple augmentation du montant de la facture mais de la part plus importante à la charge de celles et ceux qui paient les primes. Si cette part était restée constante, les primes seraient aujourd'hui 20\% moins élevées [5].

Cette évolution vers une charge croissante des primes risque de se poursuivre si les responsables politiques n'agissent pas. Lorsque nous traitons plus de patients en ambulatoire sans les hospitaliser, c'est la prise en charge médicale dans son ensemble qui devient moins chère. Or, dans le même temps, elle devient plus chère pour les payeurs de primes car ces mêmes traitements ambulatoires relèvent exclusivement des primes, à l'inverse des hospitalisations principalement financées par les impôts. Ce transfert pénalise avant tout les ménages à faible revenu, qui s'acquittent des mêmes primes par personne indépendamment de leurs capacités économiques.

Une solution susceptible de mettre fin au caractère antisocial de ce transfert, tout en permettant d'exploiter d'importantes sources d'efficacité, est entre les mains du Parlement depuis plus de dix ans [6], malheureusement sans grand résultat pour l'instant. Avec le financement uniforme des prestations ambulatoires et stationnaires (EFAS), la part relevant des primes et celle des cantons seraient fixes, indépendamment du fait que le traitement soit dispensé en ambulatoire ou exige une hospitalisation. Le potentiel des traitements ambulatoires pourrait être pleinement exploité et, surtout, les modèles de soins intégrés présentant des avantages importants en termes de coûts pour les assurés avoir de nouveau le vent en poupe. En fin de compte, une répartition plus sociale de la charge associée à des économies

Si la part de soins financée par les primes avait cessé de croître, les primes seraient aujourd'hui $20 \%$ moins élevées.

de plusieurs milliards chaque année permettrait d'alléger sensiblement la facture, alors que la qualité de la prise en charge médicale serait même améliorée.

C'est au Parlement de décider si les raisons d'être optimistes iront au-delà des seules augmentations modérées de ces dernières années. A l'inverse d'autres projets politiques, l'EFAS pourrait avoir un effet durable sur les primes, sans impacter la prise en charge médicale. Il existe depuis longtemps une large alliance des acteurs de la santé et du Konsumentenforum, tous favorables à l'EFAS [6]. Maintenant, il ne reste plus qu'aux responsables politiques à agir.

\section{Références}

La liste complète des références est disponible dans la version en ligne de l'article sur www.bullmed.ch 